\title{
Thorax mapping for localized lung impedance change using focused impedance measurement (FIM): A pilot study
}

\author{
Humayra Ferdous ${ }^{1,3}$, Tanvir Noor Baig ${ }^{2}$ and K. Siddique-e Rabbani ${ }^{2,3}$ \\ 1. Department of Physics, University of Oslo, Oslo, Norway \\ 2. Department of Biomedical Physics and Technology, University of Dhaka, Dhaka-1000, Bangladesh \\ 3.E-mail any correspondence to: humayraf@student.matnat.uio.no or rabbani@univdhaka.edu
}

\begin{abstract}
Focused impedance measurement (FIM) is a technique where impedance can be measured with the optimum level of localization without much increase in complexity of measuring instrument. The electrodes are applied on the skin surface while the organs inside also contribute to the measurement, as the body is a volume conductor. In a healthy and disease free lung region, the air enters at breathe-in, increases the impedance of the lung, and impedance reduces during breathe-out. In contrast, for a diseased lung, where part of the lungs is filled with water or some fluid, air will not enter into this zone reducing impedance change between inspiration and expiration. With this idea, the current work had been executed to have general view of localized impedance change throughout thorax using 6-electrode FIM. This generated a matrix mapping from both the front and from the back of the thorax, which showed how impedance change due to ventilation varies from frontal plane to back plane of human bodies.
\end{abstract}

Keywords: Focused impedance measurements, FIM, lung impedance, bioimpedance

\section{Introduction}

Focused impedance measurement (FIM) $[1,2]$ is a comparatively new technique that had been developed at the Department of Biomedical Physics and Technology (BMPT) of the University of Dhaka for measuring impedance. It has three versions having 8,6 and 4 electrodes in special configurations. In a 6-elctrode-FIM, current is driven through two concentric pairs of orthogonal electrodes while another pair of electrodes with a smaller separation is placed at $45^{\circ}$ to either of the current drive directions at the central zone for potential measurement. The resultant potential measurement possesses has a dominant influence from the central zone, which is nearly a square with the potential electrodes at the diagonal points. Thus it localizes the sensitivity at the central area, which has been termed the "focused zone."

FIM with its 3D sensitivity had been effective in the study of measuring gastric emptying [2]. A linear relationship to change in expired volume of air was found in a previous study when implemented to a focused zone of the lung in a subject [3]. In principle, the dielectric properties of lung tissue varies greatly as a result of air ventilation, between expiration and inspiration [4], which ultimately offers an area of opportunity to implement electrical impedance measurement systems for further study, such as for lung disease detection. However, to apply FIM for lung impedance study and disorder detection, it is essential to verify whether FIM can be applied to measure the changes of lung impedance due to ventilation in localized regions. In the current work the 6-electrode FIM had been applied to some healthy human subjects to measure the change in impedance in between full inspiration and full expiration.

\section{Materials and methods}

\section{Instrumentation}

Instrumentation for FIM developed earlier at BMPT was used for the present study. A simplified block diagram is shown in Figure 1 [2]. A sinusoidal signal, $10 \mathrm{kHz}$, is split up into two isolated current drives ( $\mathrm{AA}^{\prime}$ and $\mathrm{BB}$ '). This was done through appropriate voltage to current converters and isolating transformers. The circle in Figure 1 represents an object under test, which could be a human body. Amplitudes of each of the current drives were adjusted to have two equal perpendicular driving currents. The phases of the current drives were adjusted through the electrode connections such that the output signal at $\mathrm{u}$ and $\mathrm{v}$ of both the orthogonal drives appear in phase. Thus a single measurement of potential across $u$ and $v$ gives the sum of the combined transfer impedance in both the orthogonal directions. Subsequent amplification and signal conditioning circuitry gives a de signal that is proportional to the combined impedance. This dc output voltage was measured using a digital voltmeter.

\section{Subject selection}

The present work was performed at BMPT and volunteers were mainly students of this department, with no known acute or chronic lungs disease and with no previous complaint of respiratory problems. All of them were nonsmokers and their ages ranged from 17 to 26 years.

\section{Electrode Configuration}

The electrode arrangement was indicated in Figure 1, where electrode pairs AA' and BB' were the current drives while the potential was measured across the pair uv. In the present experiment this electrode configuration was used to 


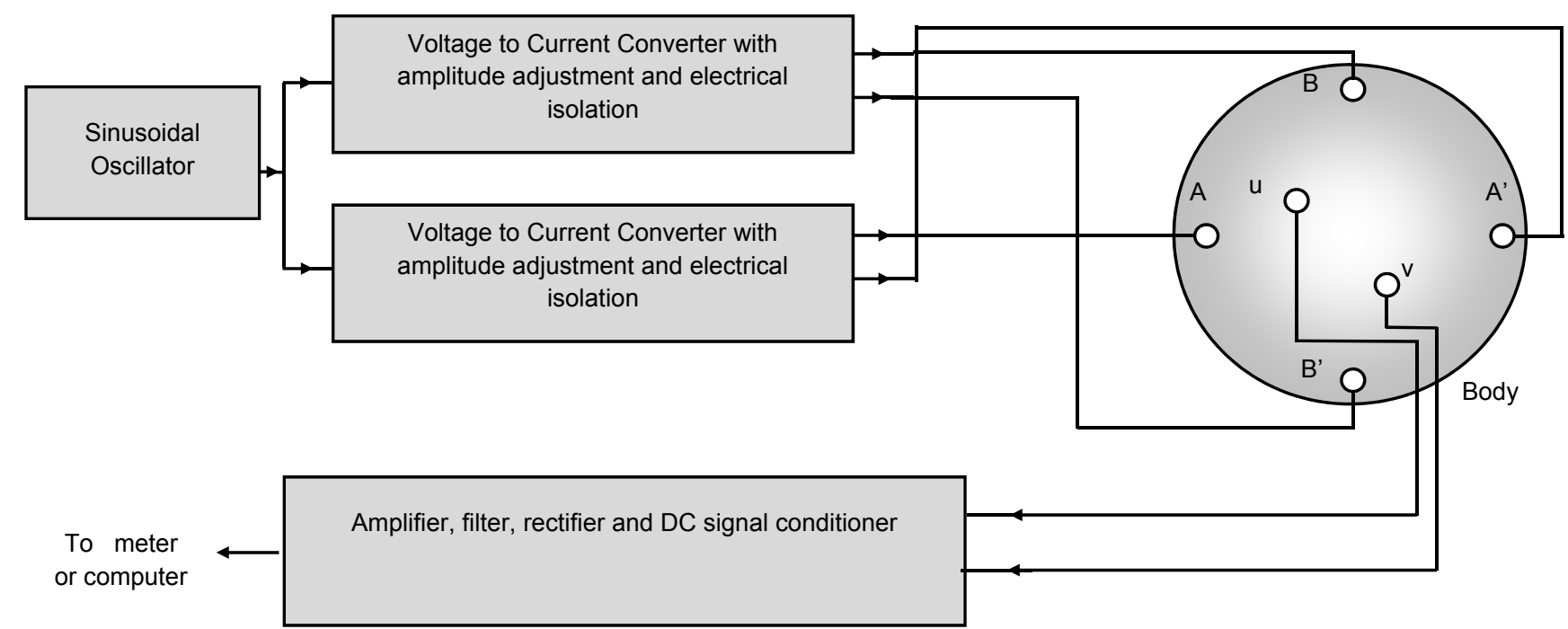

Fig. 1: A block diagram of the 6-electrode FIM instrumentation

map the lungs both from the front and from the back of the thorax. In this electrode configuration the focused region is defined by a square with uv as the diagonal, shown shaded in Figure 2.

To map consecutive focused zones on the thorax we placed the electrodes in a 'diamond' configuration, as shown in Figure 2, since the focused zone is tilted at $45^{\circ}$ to the sides of the larger square formed by the current drive electrodes. As shown, the sides of the larger square were 4 inches $(\sim 10 \mathrm{~cm})$ while the diagonal of the smaller square was 2 inches $(\sim 5 \mathrm{~cm})$, corresponding to sides of about 1.4 inches $(\sim 3.5 \mathrm{~cm})$. The measurement required a common reference electrode, which was placed at the center (small black circle). The colors red and black are used for the two current drive pairs of electrodes in Figure 2 to indicate the relative phases (red indicating positive polarity at an instant). Figure 3 shows a picture of the hand held electrode probe used. Wet cotton wool is inserted into cylindrical

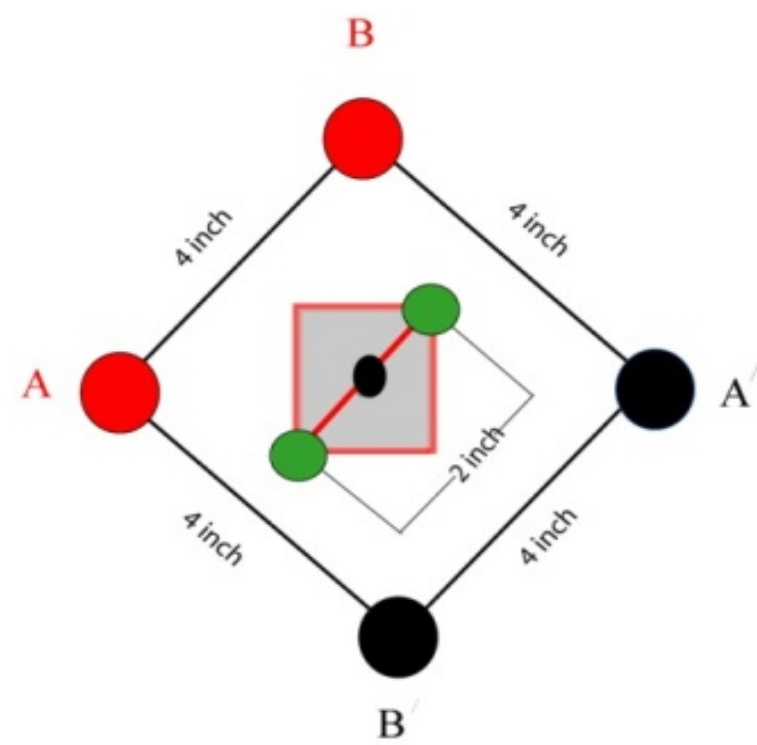

Fig. 2: The schematic diagram of 'diamond' type electrode configuration for the 6-electrode FIM system, the shaded square region bounded by red color represents the focused zone. recesses touching the metallic electrode inside an insulated cylindrical structure. Each electrode has a spring-loaded stem allowing perpendicular movement in order to ensure good connections at curved body surfaces.

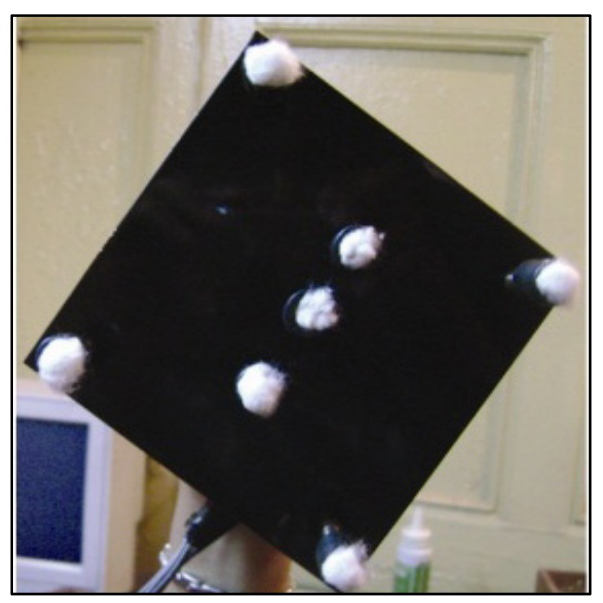

Fig. 3: The handheld electrode probe, seen from the electrode side. Wet cotton wool is inserted into recesses touching the metallic electrode inside. The stems of the electrodes are springloaded to ensure good contact at curved body surfaces.

\section{Body mapping Matrix}

As mentioned above, the side of the focused region is about 1.4 inches $(\sim 3.5 \mathrm{~cm})$. Therefore, to obtain the lung impedance map at successive square regions in a matrix as shown in Figure 4, we moved the electrode by the same distance horizontally and vertically, as indicated in Figure 5. To facilitate measurement on human subjects guiding points were marked out on the front and back of the thorax using a paper template as shown in the photographs of Figure 6. The cell numbers shown in the last two photographs were superimposed in computer later; these were not marked on the human subjects. Thus a standard design was developed so that the impedance changes at individual matrix locations can be compared among subjects of similar body size. Figure 4 also shows the 
reference matrix numbers that were used in the analyses presented later.

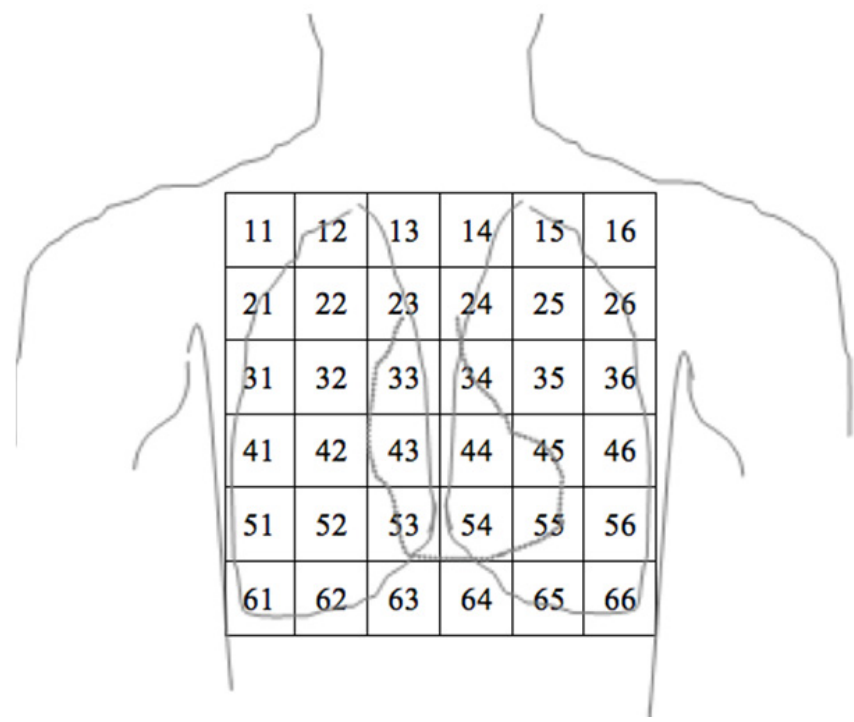

Fig. 4: The desired body-mapping matrix with values of impedance. The measurements are performed both from the front and the back.

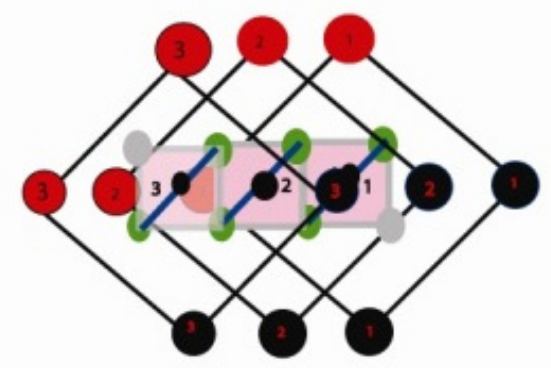

Fig. 5: Movement of hand held electrode probe to obtain the desired body mapping matrix.

\section{Measurement of ventilation}

Measurements were made with the subject standing straight. The output voltage of the FIM system, which was measured using a digital multi-meter, is proportional to the impedance of the focused region defined before. For each position of the electrode, a reading of the focused impedance was taken with the subject breathing in fully (forced maximum inspiration) and another reading with the subject breathing out as much as possible (forced maximum expiration). The difference was expressed as a percentage of the value at forced maximum expiration at that location. Measurements were taken from both the front and the back according to the matrix described before. Figure 7 shows typical measurement procedures from the front and from the back. The spring-loaded electrode stem allowed a good contact even at curved regions. The total volume of air between forced maximum inspiration and forced maximum expiration is known as forced vital capacity (FVC), which was measured using a bellows type spirometer.

\section{Results}

The results of the study on four subjects are presented in Table 1. The left major column shows the localized percentage change in impedance in each matrix location measured from the front of the thorax while the right major column shows the same as measured from the back. The right-left orientation of the measured values from the back was rearranged in the matrices to match that from the front, to enable a simple visual comparison. The age, height, weight, and forced vital capacity of the subjects are all indicated in the tables.

The values at each matrix element are assumed to correspond to localized ventilation within that region. It needs to be appreciated that the measured impedance values have the major contribution from the soft tissue underlying the skin, which does not change with ventilation. The depth sensitivity of the FIM decreases sharply with depth $[5,6]$, therefore, a small contribution comes from the ventilating lungs. Subtraction of the two values of impedance on inspiration and on expiration accentuated the ventilation, which was the justification of these attempts at measurement of ventilation.

Table 2 shows the comparison between the impedance percentages changes at frontal plane and backplane of the human bodies. However, the measured values near the edges were expected to be erroneous, because of two reasons: i) large curvatures at the sides making it difficult, even impossible for all electrodes of the probe to touch the skin properly, and ii) the movement of the edges of the expanding and contracting lungs falling within the measured regions, contributing to the measurement significantly. Therefore, the central matrix elements numbered 22 to 45 were considered for the present analysis.

Table 3 was developed to represent the comparison of impedance percentage change between the right side and the left side of each plane. In this particular comparison column 2 and 3 of each plane were considered the right side of the body whereas column 4 and 5 were considered the
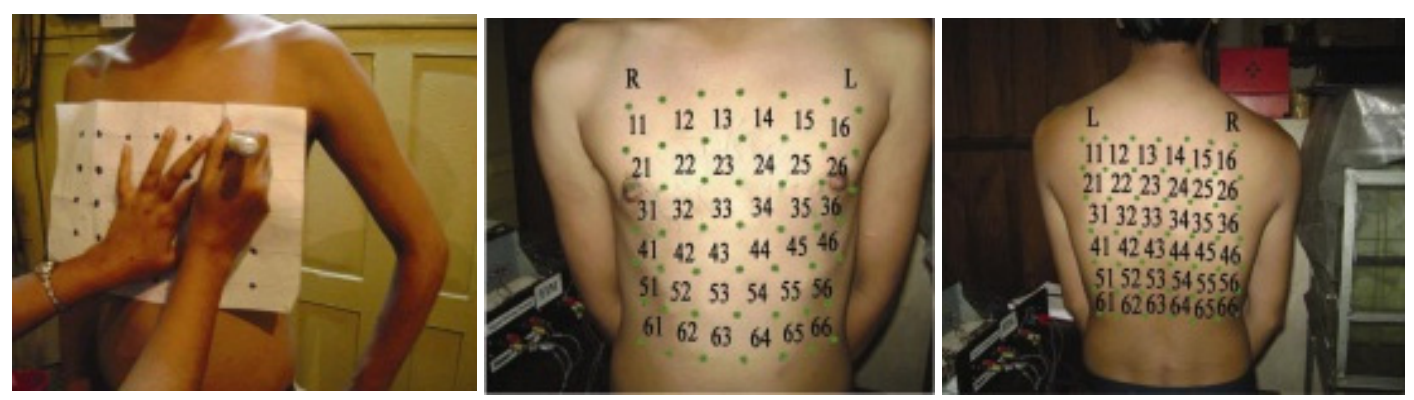

Fig. 6: Technique for marking out identification of points on thorax for the desired matrix for measurement. The cell numbers shown were superimposed later on the photographs. 
left side of the body leaving out 1 and 6 column at each plane due to it erroneousness, as mentioned earlier. The second row in each plane is deemed "top" in Table 3. Similarly, the third and fourth rows of each plane are referred to as "mid" and "low," respectively in Table 3, for understanding the comparison at a glance.

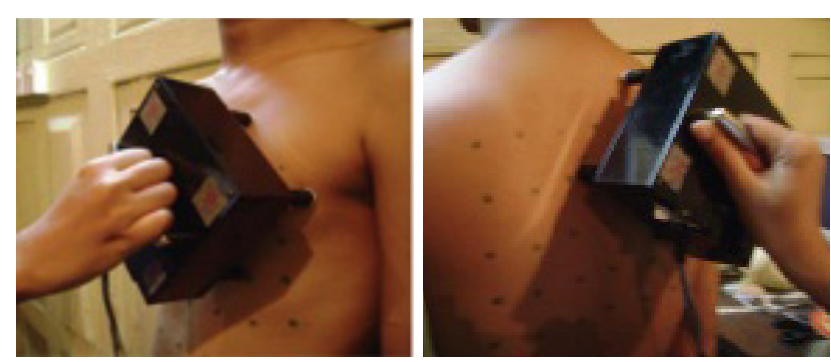

Fig. 7: Measurement of localized ventilation using the hand held probe with spring loaded electrodes.

Table 1: Percentage change in localized impedance in each individual matrix, as measured from the front and from back. The right-left orientation are arranged to be the same on both views, for easy visual comparison.

\begin{tabular}{|c|c|c|c|c|c|c|c|c|c|c|c|}
\hline \multicolumn{6}{|c|}{ Measurement from Front } & \multicolumn{6}{|c|}{ Measurement from Back } \\
\hline \multicolumn{2}{|c|}{ Right } & \multicolumn{4}{|c|}{ Left } & \multicolumn{3}{|c|}{ Right } & & \multicolumn{2}{|c|}{ Left } \\
\hline \multicolumn{12}{|c|}{ Subject-1: age: $26 \mathrm{y}$, Height: $179 \mathrm{~cm}$, Weight: $67 \mathrm{~kg}, \mathrm{~F}$ Vital capacity: $3.8 \mathrm{~L}$} \\
\hline 3.5 & 6.7 & 13.9 & 9.5 & 3.3 & 1.9 & 2.6 & 7.6 & 1.8 & 1.9 & 2.2 & 1.5 \\
\hline 0 & 6.8 & 11.6 & 5.5 & 8.5 & 0 & 1.8 & 2.8 & 2.1 & 6.1 & 1.2 & 1.7 \\
\hline 14.1 & 8.2 & \begin{tabular}{|l|}
6.5 \\
\end{tabular} & 5.5 & 7.1 & 6.4 & 3.9 & 2.5 & \begin{tabular}{|l|}
1.9 \\
\end{tabular} & 2.1 & 2.6 & 3.3 \\
\hline 0 & 6.5 & \begin{tabular}{|l|}
5.8 \\
\end{tabular} & 8.6 & 6.7 & 0 & 0 & 2.2 & 1.3 & 4 & 3.2 & 6. \\
\hline 0 & \begin{tabular}{|l|}
4.3 \\
\end{tabular} & 2.8 & 4.7 & 2.1 & 0 & 0 & 3.3 & 2.7 & 2.2 & 4.7 & 0 \\
\hline 4.1 & 0 & 2.7 & 0 & 3.9 & 7.1 & 0 & 0 & 3.4 & 2.2 & 3.8 & 0 \\
\hline \multicolumn{12}{|c|}{ Subject-2: age: 18 y, Height: $174 \mathrm{~cm}$, Weight: $70 \mathrm{~kg}, F$ Vital capacity: $4.4 \mathrm{~L}$} \\
\hline 7.7 & 5.3 & 15.1 & 6.8 & 4.8 & 2.4 & 3.8 & 7.8 & 4.8 & 2.6 & 4.6 & 7.6 \\
\hline 8.1 & 11.7 & 14.3 & 6.7 & 4.5 & 3.8 & 1.5 & 1.6 & 4.4 & 7.2 & 4.4 & 7.6 \\
\hline 11.3 & 15 & 16.7 & 12.4 & 13.6 & 6.3 & 3.3 & 10.7 & 4.8 & 5 & 10.5 & 6 \\
\hline .5 & 12.1 & 10.6 & 4.8 & 12.7 & 20.4 & 9.4 & 7.1 & 3.3 & 4.3 & 3.6 & 9.5 \\
\hline 19.1 & 12.9 & 4.1 & 8.2 & 12.4 & 16 & 14.1 & 6 & 4.3 & 3.3 & 3.7 & 5.8 \\
\hline \multicolumn{12}{|c|}{ Subject-3: age: 26 y, Height: $169 \mathrm{~cm}$, Weight: $65 \mathrm{~kg}, F$ Vital capacity: $3 \mathrm{~L}$} \\
\hline 8.5 & 10.9 & 10.5 & 10.5 & 12.8 & 8.8 & 0 & 0 & 0 & 0 & 0 & 0 \\
\hline 14.9 & 13.3 & 30.6 & 14.6 & 6.3 & 0 & 6.3 & 7.4 & 11.1 & 3.8 & 13.2 & 12.7 \\
\hline 0 & 14.8 & 15.9 & 15.2 & 14.5 & 15.4 & 8.2 & 15.4 & 5.7 & 3.6 & 5 & 9.7 \\
\hline 12.3 & 12.2 & 12.5 & 10.1 & 7.3 & 15.5 & 5.4 & 8.3 & 3.7 & 4.5 & 4.7 & 10 \\
\hline 21.1 & 17.2 & 12 & 8.1 & 26.2 & 21.7 & 4.8 & 2 & 0 & 0 & 7.3 & 8.9 \\
\hline 4.5 & 12 & 7.7 & 5.3 & 2 & 10.6 & 0 & 0 & 0 & 0 & 0 & 0 \\
\hline \multicolumn{12}{|c|}{ Subject-4: age: $20 y$, Height: $172 \mathrm{~cm}$, Weight: $74 \mathrm{~kg} F$ Vital capacity: $3 \mathrm{~L}$} \\
\hline 2 & 2.6 & 4.2 & 7.1 & 2.8 & 1.1 & 1.6 & 1 & 3 & 3.1 & 2.8 & 1.2 \\
\hline 4.5 & 3 & 7.2 & 3.6 & 1.7 & 6 & 6.8 & 2.2 & 2.3 & 2.7 & 1.7 & 1.7 \\
\hline 5.6 & 4.7 & 8.6 & 3.1 & 1.7 & 6 & 1.6 & 0.9 & 2.6 & 4 & 4.1 & 2.3 \\
\hline 2.7 & \begin{tabular}{|l}
4.4 \\
\end{tabular} & 5.7 & 3.8 & 4.2 & 2.2 & 0.9 & 1.3 & 2.1 & 4.6 & 3.5 & 6.1 \\
\hline 4.9 & 2.14 & 1.97 & 3.6 & 5.7 & 4.8 & 2.3 & 0.9 & 0.8 & 4.2 & 5.3 & 3 \\
\hline 1.8 & \begin{tabular}{|l}
4.7 \\
\end{tabular} & 2.6 & 0 & 0 & 0 & 5 & 1.8 & 2.5 & 3.9 & 1.2 & 1.7 \\
\hline
\end{tabular}

After summing up the impedance percentage change of column 2 and 3 for each level [top $(22,23)$, mid $(32,33)$, and low $(42,43)]$, the values for right $(\mathrm{Rt})$ in the Table 3 were obtained. A similar process is followed for achieving left (Lt) values in Table 3. In the former case matrix elements 24 and 25 are used in summing up impedance percentage change at top level of each plane. For mid-level, the impedance percentage change of matrix elements 34 and 35 are summed up and for the low level, matrix elements 44 and 45 are taken into account for each plane.
The ratio between $\mathrm{Rt}$ and $\mathrm{Lt}$ for each level of each plane was calculated. The mean value of these ratios for frontal and backplane gives an idea how impedance varies from right to left side of each plane.

Table 2: Comparison of summed values from front and back for 12 matrix elements, 22 to 45 .

\begin{tabular}{|c|c|c|c|c|c|}
\hline Subject & Location & $\begin{array}{c}\text { Sum of } \\
\text { change in } \\
\text { Impedance } \\
(\%)\end{array}$ & $\begin{array}{c}\text { Forced } \\
\text { Vital } \\
\text { Capacity } \\
\text { (FVC) L }\end{array}$ & Sum/FVC & $\begin{array}{c}\text { Ratio } \\
\text { front/back }\end{array}$ \\
\hline \multirow{2}{*}{ Subj-1 } & front (elem. 22-45) & 87.3 & \multirow{2}{*}{3.8} & 23 & \multirow{2}{*}{2.73} \\
\hline & back (elem.22-45) & 32 & & 8.4 & \\
\hline \multirow{2}{*}{ Subj-2 } & front (elem.22-45) & 135.1 & \multirow{2}{*}{4.4} & 30.7 & \multirow{2}{*}{2.02} \\
\hline & back (elem.22-45) & 66.9 & & 15.2 & \\
\hline \multirow{2}{*}{ Subj-3 } & front (elem.22-45) & 167.3 & \multirow[b]{2}{*}{3} & 55.7 & \multirow{2}{*}{1.94} \\
\hline & back (elem.22-45) & 86.35 & & 28.7 & \\
\hline \multirow{2}{*}{ Subj-4 } & front (elem.22-45) & 51.7 & \multirow{2}{*}{3} & 17.2 & \multirow{2}{*}{1.61} \\
\hline & back (elem.22-45) & 32 & & 10.67 & \\
\hline \multicolumn{6}{|c|}{ ront/back ratio $=2.1 \pm 0.47$} \\
\hline
\end{tabular}

Table 3: Comparison of summed values between right and left sides, for three horizontal levels for 12 matrix elements, 22 to 45 of front plane and back plane

\begin{tabular}{|c|c|c|c|c|c|c|c|c|c|}
\hline \multirow[b]{2}{*}{ Subject } & \multirow[b]{2}{*}{ Location } & \multicolumn{4}{|c|}{ Frontal Plane } & \multicolumn{4}{|c|}{ Back Plane } \\
\hline & & $\begin{array}{c}\text { Right (Rt) } \\
\text { [Column } \\
2 \text { and 3] }\end{array}$ & $\begin{array}{l}\text { Left (Lt) } \\
\text { [Column } \\
4 \text { and 5] }\end{array}$ & $\begin{array}{c}\text { Ratio, } \\
\mathrm{Rt} / \mathrm{Lt} \\
\mathrm{F}\end{array}$ & $\begin{array}{c}\text { Mean } \\
\text { F }\end{array}$ & $\begin{array}{c}\begin{array}{c}\text { Right } \\
\text { (Rt) } \\
\text { [Column } \\
2 \text { and } 3]\end{array} \\
\end{array}$ & $\begin{array}{l}\text { Left (Lt) } \\
\text { [Column } \\
4 \text { and } 5 \text { ] }\end{array}$ & $\begin{array}{c}\text { Ratio, } \\
\text { RtLt } \\
\text { B }\end{array}$ & Mean B \\
\hline \multirow{3}{*}{ Subject 1} & $\begin{array}{l}\text { top } \\
\text { (elem. 22-25) }\end{array}$ & 18.4 & 14 & 1.31 & \multirow{12}{*}{$1.55 \pm 0.6$} & 4.9 & 7.3 & 0.67 & \multirow{12}{*}{$0.96 \pm 0.6$} \\
\hline & $\begin{array}{c}\text { mid } \\
\text { (elem. 32-35) }\end{array}$ & 14.7 & 12.6 & 1.17 & & 4.4 & 4.7 & 0.94 & \\
\hline & $\begin{array}{c}\text { low } \\
\text { (elem. } 42-45 \text { ) }\end{array}$ & 12.3 & 15.3 & 0.80 & & 3.5 & 7.2 & 0.49 & \\
\hline \multirow{3}{*}{ Subject 2} & $\begin{array}{c}\text { top } \\
\text { (elem. 22-25) }\end{array}$ & 26 & 11.2 & 2.32 & & 6 & 11.6 & 0.52 & \\
\hline & $\begin{array}{c}\text { mid } \\
\text { (elem. 32-35) }\end{array}$ & 31.7 & 26 & 1.22 & & 15.5 & 15.5 & 1.00 & \\
\hline & $\begin{array}{c}\text { low } \\
\text { (elem. } 42-45 \text { ) }\end{array}$ & 22.7 & 17.5 & 1.30 & & 10.4 & 7.9 & 1.31 & \\
\hline \multirow{3}{*}{ Subject 3} & $\begin{array}{c}\text { top } \\
\text { (elem. 22-25) }\end{array}$ & 43.9 & 20.9 & 2.10 & & 18.5 & 17 & 1.09 & \\
\hline & $\begin{array}{c}\text { mid } \\
\text { (elem. 32-35) }\end{array}$ & 30.7 & 29.7 & 1.03 & & 21.1 & 8.6 & 2.45 & \\
\hline & $\begin{array}{c}\text { low } \\
\text { (elem. } 42-45 \text { ) }\end{array}$ & 24.7 & 17.4 & 1.42 & & 11 & 9.2 & 1.19 & \\
\hline \multirow{3}{*}{ Subject 4} & $\begin{array}{c}\text { top } \\
\text { (elem. 22-25) }\end{array}$ & 10.2 & 5.3 & 1.92 & & 4.5 & 4.4 & $\begin{array}{l}1.02 \\
\end{array}$ & \\
\hline & $\begin{array}{c}\text { mid } \\
\text { (elem. 32-35) }\end{array}$ & 13.3 & 4.8 & 2.77 & & 3.5 & 8.1 & 0.43 & \\
\hline & $\begin{array}{c}\text { low } \\
\text { (elem. 42-45) }\end{array}$ & 10.1 & 8 & 1.26 & & 3.4 & 8.1 & 0.41 & \\
\hline
\end{tabular}

\section{Discussion}

Primarily, this study was carried out to have a generalized view of localized impedance change across the thorax of human body due to breathing using 6-electrode FIM system. The ultimate objective was to employ the noninvasive FIM to detect lung disease based on the idea that the change in impedance measurement of diseased lung during breathe in and breathe out would be significantly less compared to that of a healthy one since air content will be reduced by the presence of water, blood, fluid, other substance or any other injury. This indication can only be functional and influential when the study on the large number of normal lungs takes place and a standard set of values are available to compare with the diseased lungs of different category. Furthermore the changes in impedance at different region at each plane (both front and back) are also dependent on anatomical structure of the lung as well as the body structure of the subjects, which led to use at times $6 \times 6$ matrix or $6 \times 5$ matrix.

It is noticeable from the Table 1 that the changes in impedance are greater when it is measured from the anterior plane than that of the posterior plane. For the Subject-1 in 
Table 1, the maximum change occurred in the frontal plane $(14.1 \%)$ with a second maximum change of $13.9 \%$, whereas the maximum change in impedance in the back plane for the same subject was $7.6 \%$ with a second maximum of $6.1 \%$.

For the next subject (Subject-2 in Table 1), the maximum change of impedance was $20.4 \%$ and a second maximum change of impedance is $19.1 \%$ for frontal plane, whereas when measured from the back the maximum and the second maximum change in impedance shrunk to $14.1 \%$ and $10.7 \%$, respectively.

Analyzing Subject-3 in Table 1, it was found that the maximum change of impedance was $30.6 \%$ and the second maximum change of impedance was $26.2 \%$ for the frontal plane. When it was measured from the back, the maximum and the second maximum change in impedance were $15.4 \%$ and $13.2 \%$, respectively.

Subject-4 in Table 1 also supports us in this conclusion, as the maximum change of impedance in this subject was $8.6 \%$ with a second maximum change of impedance of $7.2 \%$ for the frontal plane. When it was measured from the back, the maximum and the second maximum change in impedance became $6.8 \%$ and $6.1 \%$, respectively. This may be because the sensitivity of the focused zone decreases with depth.

Moreover, the right most and the left most column at each plane usually possess larger values compared to the other column. Here also the change in impedance $(\%)$ in frontal plane remained higher than that of the back plane. One of the reasons for this could be due to the presence of solid bones right under these focused zones. Differences in vital capacity for each subject should be taken into account too. The human error of in regards to the instructions of full expiration and full inspiration may also cause to this result.

There are some cases when negative values do occurthough not considered in this study to avoid complication and are needed to develop advanced FIM applications. The change in impedance at lower side (the bottom most row in each matrix) of the lungs of each plane varies abruptly in some cases such as the frontal and backplane of Subject-2 and back plan of Subject-4 in Table 1. The possible reason could be the diaphragm of the lungs, which expands and shrinks due to ventilation in this region and contributes to the impedance change to great extent.

In addition, it is seen from Table 2 that the average ratio between total impedance percentage change of the frontal plane and back plane is 2.1 (Table 2) i.e., on average the total impedance percentage changes at the frontal plane is twice as much as that of the backplane for each subject. This leaves another field to explore with more detail approach.

The potential reason for such higher values of impedance percentage change at the front plane might be the existence of heart at the frontal plane. The matrix column 2, 3, 4, 5 at frontal plane resembles cardiac region of human body and from Table 1 it is seen that the impedance percentage change in these focused zones varied significantly, especially when measured from the front, as mentioned earlier. So the ratio value from Table 2 also insinuates how heart and solid ribs contribute to the impedance change, though experiments with larger number of subjects are required to establish this result.

From Table 3, it is found that the impedance percentage change of the right side of frontal plane of human body is $\sim 1.6$ times that of left side of the same plane. The same value shrinks to $\sim 1$ when the comparison of right and left side of back plane is considered. The possible reason for such difference might be the same as mentioned earlier, i.e., contribution of heart at frontal plane and existence of muscle at back plane.

Nevertheless, it becomes more critical for the female subjects in terms of impedance change measurement due to the presence of fat tissue on anterior plane of thorax providing the same result found when the change in impedance was measured using the dice or diamond shape localized zone [7].

\section{Conclusion}

This present work has enhanced our understanding on lung impedance change due to ventilation with relatively simple and low-cost instrumental FIM setup. Consequently, advance study in this direction should explore different aspects and features.

\section{Acknowledgement}

The authors would like to express their gratitude to the volunteers for their participation in the study.

\section{References}

1. Rabbani KS, Sarker M, Akond MHR and Akter T. Proceeding of the X International Conference on Electrical Bioimpedance, Barcelona, Spain. 1998:31-34.

2. Rabbani KS, Sarker M, Akond MHR, Akter T. Ann. N.Y. Acad. Sci. 1999;873:408-420. http://dx.doi.org/10.1111/j.1749-6632.1999.tb09490.x

3. Rabbani KS, Kadir MA. Possible applications of Focused Impedance Method (FIM) in biomedical and other areas of study. Bangladesh J. Med. Phys. 2011;4(1):67-74.

4. Nopp P, Rapp E, Pfutzner H, Nakesch H and Ruhsam C. Phys. Med. Biol. 1993;38:699-719. http://dx.doi.org/10.1088/0031-9155/38/6/005

5. Islam, N, Rabbani, KS, Wilson A. The sensitivity of focused electrical impedance measurements. Physiol. Meas. 2010;31: S97-109. http://dx.doi.org/10.1088/0967-3334/31/8/S08

6. Iquebal AH Masum, Rabbani KS. 3D sensitivity of 6-electrode Focused Impedance Method. J. Phys. Conf. Ser. 2010;224:012156. http://dx.doi.org/10.1088/1742-6596/224/1/012156

7. Kadir MA, Ferdous H, Baig TN, Rabbani KS 2010. Ventilation mapping of chest using Focused Impedance Method (FIM). J. Phys. Conf. Ser. 2010;224: 012031. http://dx.doi.org/10.1088/1742-6596/224/1/012031 